\title{
How successful is liver resection for colorectal cancer liver metastases in patients over 75 years old?
}

\author{
Nicholas George Mowbray ${ }^{1}$, Carven Chin ${ }^{2}$, Patricia Duncan ${ }^{1}$, David O'Reilly ${ }^{1}$, \\ Zsolt Kaposztas ${ }^{3}$, Sameer Junnarkar ${ }^{4}$, and Nagappan Kumar ${ }^{1}$ \\ ${ }^{1}$ Cardiff Liver Unit, University Hospital of Wales, ${ }^{2}$ Cardiff University School of Medicine, Cardiff, UK, \\ ${ }^{3}$ Department of Surgery, Moritz Kaposi Teaching General Hospital, Kaposvarr, Hungary, \\ ${ }^{4}$ Department of Surgery, Tan Tock Seng Hospital, Singapore
}

\begin{abstract}
Backgrounds/Aims: As populations age, an increased incidence of colorectal cancer will generate an increase in colorectal cancer liver metastases (CRLM). In order to guide treatment decisions, this study aimed to identify the contemporary complication rates of elderly patients undergoing liver resection for CRLM in a, centralised, UK centre. Methods: All patients undergoing operative procedures for CRLM between January 2013 and January 2019 were included. Patient, tumour and operative data were analysed, including the prognostic marker; tumour burden score. Results: 339 operations were performed on 289 consecutive patients with CRLM (272 patients < 75 years old, 67 patients $\geq 75$ years old). Median age was 66 years (range 20-93). There was no difference in major complication rates between the two age cohorts $(6.65$ vs. $6.0 \%, p=0.847)$ or operative mortality $(1.1 \%$ vs. $1.4 \%, p=0.794)$. Younger patients had higher R1 resection rates $(20.4 \%$ vs. $4.5 \%, p=0.002)$ and post-operative chemotherapy rates $(60.3 \%$ vs. $35.8 \%, p<$ 0.001 ). The 1,3 and 5 -year OS was $90.2 \%, 70.5 \%$ and $52.3 \%$ respectively, median 70 months, with no difference between age cohorts $(p=0.772)$. Tumour Burden score and operation type were independent predictors of overall survival. Conclusions: Liver resection for CRLM in patients 75 years and older is feasible, safe and confers a similar 5-year survival rate to younger patients. The current outcomes from surgery are better than historical datasets. (Ann Hepatobiliary Pancreat Surg 2021;25:18-24)
\end{abstract}

Key Words: Liver; Colonic neoplasms; Neoplasm metastasis

\section{INTRODUCTION}

The largest risk factor for developing colorectal cancer is increasing age and estimates suggest the population aged 75 years and older is estimated to almost double to 9.8 million over the next 25 years in the UK. ${ }^{1,2}$ Approximately $25 \%$ of patients with colorectal cancer will develop liver metastases within 5 years, and surgical resection offers patients the greatest chance of long-term survival. ${ }^{3}$ The net effect, therefore, is likely to be an increase in elderly patients requiring liver resections. ${ }^{4}$

The existing literature on the resection colorectal cancer liver metastases (CRLM) often uses historical data to show a higher operative morbidity, less aggressive surgery and a reduced overall survival in patients aged over 75 years old. ${ }^{5}$ Developments in the operative, and peri-operative, management of patients undergoing liver resection is likely to have improved outcomes and up to date data is required.

This study was undertaken to identify the contemporary morbidity and mortality rates for patients 75 years or older undergoing liver resection for CRLM in a UK centre. The primary hypothesis was that there is no difference in the post-operative outcomes of patients under 75 years old compared to those older.

\section{MATERIALS AND METHODS}

\section{Study population}

All patients undergoing a liver resection for CRLM at

Received: July 1, 2020; Revised: August 30, 2020; Accepted: September 1, 2020

Corresponding author: Nagappan Kumar

Cardiff Liver Unit, University Hospital of Wales, Cardiff CF14 4XW, UK

Tel: +44(0)2920744793, Fax: +44(0)2920743348, E-mail: nagappan.kumar@wales.nhs.uk

Copyright (C) 2021 by The Korean Association of Hepato-Biliary-Pancreatic Surgery

This is an Open Access article distributed under the terms of the Creative Commons Attribution Non-Commercial License (http://creativecommons.org/ censes/by-nc/4.0) which permits unrestricted non-commercial use, distribution, and reproduction in any medium, provided the original work is properly cited. Annals of Hepato-Biliary-Pancreatic Surgery • pISSN: 2508-5778 - eISSN: 2508-5859 
the University Hospital of Wales (Cardiff, UK) across a 6-year period, $1^{\text {st }}$ January 2013 until $1^{\text {st }}$ January 2019, were included. Patients were excluded if post-operative histology identified an alternative pathology. Operative procedures were performed with the intention of curative liver resection after discussion at a regional specialist multidisciplinary team meeting (MDT). There was no rigid protocol for the use of primary first surgery or neo-adjuvant therapy, but rather this was assessed on a case by case basis. Operative strategies utilised open and laparoscopic procedures. If the future liver remnant was considered inadequate either Portal vein embolization (PVE) or partial ALPPS (Associating Liver Partition and Portal vein ligation in staged hepatectomy) was performed. ${ }^{6}$ Patients were routinely managed for the first post-operative night in the post anaesthetic care unit, a level 2 care environment, before returning to a ward.

\section{Data collection}

Clinicopathological and operative data were obtained from both a prospectively maintained database, and retrospectively from electronic patient databases. Data was anonymised and included baseline characteristics (American Society of Anaesthesiologists class (ASA), gender, age, chemotherapy exposure, extrahepatic disease) operative variables (extent of liver resection, intraoperative blood loss), tumour variables (margin status, bile duct involvement, vascular involvement, number of metastases, size of largest metastases), post-operative complications, disease recurrence and date of death. The tumour burden score (TBS) was developed by Sasaki et al. ${ }^{7}$ as a prognostic marker. This was calculated where $\mathrm{TBS}^{2}=(\text { maximum tumour diameter })^{2} \times$ (number of liver lesions) ${ }^{2}$.

Comparisons between the study groups were assessed with the student t-test for continuous variables, and the chi-square test for categorical variables. The Kaplan-Meier method was used to calculate survival estimates and comparisons made with the log rank test. Univariate and Multivariate regression analysis was performed using the Cox Proportional Hazards model. All statistical calculations were undertaken using SPSS Version 26 (IBM Corp. Armonk, NY). A $p$-value less than 0.05 was considered statistically significant.

\section{Definitions}

The CRLM was classified as synchronous disease if identified at, or before, the time of surgery for the primary colorectal cancer. Liver metastases presenting within 12 months or after 12 months after the colorectal surgery were considered early and late metachronous disease respectively.

Neoadjuvant and adjuvant chemotherapy refers to treatment before and after the liver surgery, not the primary colorectal surgery. Patients were classed as receiving chemotherapy if they started treatment. Resection margin positivity (R1) was defined as microscopic tumour within 1 $\mathrm{mm}$ of the margin on any specimen. Recurrent hepatic disease that occurred simultaneously with extra-hepatic disease was coded as a hepatic recurrence for analysis purposes.

Patients were followed-up for 10 years or until a clinician determined further treatments would be inappropriate. Patients were seen annually for a clinical review, serum tumour markers and a CT thorax, abdomen and pelvis to identify treatable recurrent disease.

\section{RESULTS}

\section{Clinical characteristics and operative outcomes}

Across the study period, 339 operative liver procedures were performed on 289 consecutive patients for CRLM. The median age was 66 years (range 20-93), the median ASA was 2 and $66.1 \%$ of patients were males. The majority of patients had synchronous CRLM (54.9\%), compared with $14.1 \%$ early and $30.0 \%$ late metachronous disease. The median length of stay for all procedures was 7 days with a 30 -day and 90-day mortality of $1.4 \%(4 / 289)$ and $2.4 \%(7 / 289)$ respectively. Four deaths were for liver related complications and 3 patients were discharged home well but died of a cardiac event within 90 days of surgery.

The most common procedure was a liver metastasectomy (44.2\%). Sixteen patients underwent a partial ALPPS to treat CRLM and of these, 4 patients did not return for a second stage due to disease progression and were excluded form survival estimate analysis. A further 8 patients underwent their first liver resection for CRLM prior to 2013 and therefore were also excluded. Fig. 1 summarises the patient procedures during the study period and those included in survival analysis. The 1, 3 and 5-year OS of 277 
patients was $90.2 \%, 70.5 \%$ and $52.3 \%$ respectively, with a median survival of 70 months.

\section{Age cohort analysis}

A total of 67 operations (19.8\%) were performed on patients aged 75 years and older. The OS for those under 75 years was not statistically different from those over 75 years old ( $p=0.772$, median 70 vs. 60 months) and is displayed in Fig. 2A. The 1, 3 and 5-year survival rates were $90.6 \%, 70.4 \%, 53.4 \%$ vs. $88.8 \%, 70.9 \%, 47.6 \%$ respectively. The patient characteristics of the two age groups are compared in Table 1. There was a lower rate of $\mathrm{R} 1$ resection in the older age group $(20.4 \%$ vs. $4.5 \%, p=0.02)$ as well as a lower adjuvant chemotherapy rate (60.3 vs. $35.8 \%$, $p<0.001$ ).

The median total number of tumours resected per patient was 2 in both age cohorts but ranged from 1 to 20

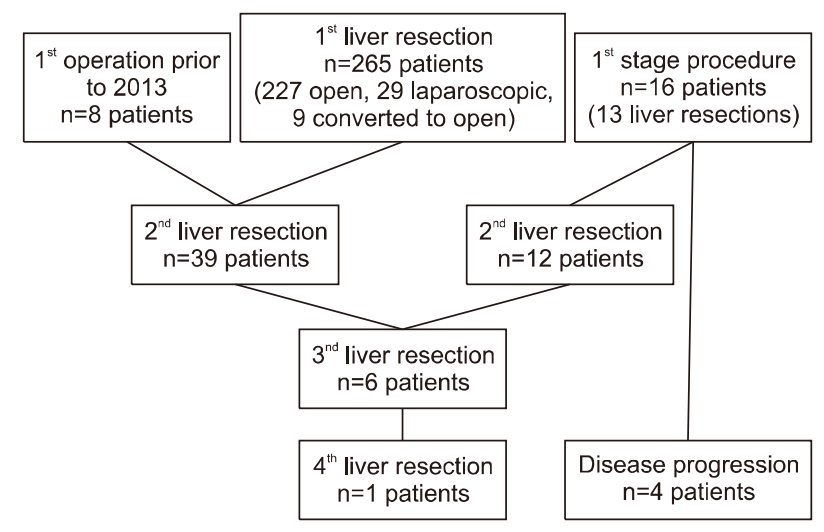

Fig. 1. Flowchart illustrating patient inclusion in the study.

A

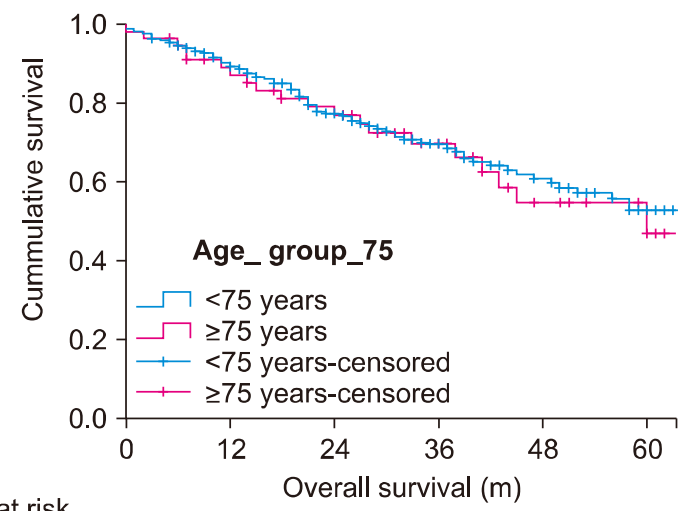

Number at risk

$\begin{array}{lllllll}<75 \text { years } & 221 & 184 & 133 & 91 & 53 & 29\end{array}$ in those aged $<75$ years and 1 to 9 in those $\geq 75$ years $(p=0.383)$.

In patients who underwent curative liver resection in the absence of extra-hepatic disease, the disease-free survival was not different in those $<75$ years vs. those greater than or equal to 75 years old $(1,3$ and 5 -year survival rates $68.7 \%, 43.7 \%$ and $41.2 \%$ vs. $80.8 \%, 43.3 \%$ and $34 \%, p=0.521)$.

\section{Prognostic factors analysis}

Univariate analysis of patient and tumour characteristics showed age $>75$ years did not influence OS $(p=0.773)$. Table 2 indicates the prognostic ability of other clinicopathological factors. Operation type $(p=0.010)$, greater TBS $(p=0.006)$ and the presence of synchronous extra-hepatic disease $(p=0.027)$ were associated with decreased OS. In multivariate analysis, both operation type and TBS remained predictive of OS (Table 3 ).

\section{DISCUSSION}

Liver resection for the treatment of metastatic colorectal cancer has developed over recent decades to the extent that patients aged 93 years old can undergo the procedure safely. The observed low morbidity rates in liver surgery are likely to result from improvements in both perioperative anaesthetic care and operative techniques (e.g. laparoscopic and parenchymal sparing). However, surgery should not be performed simply because it is technically possible, but rather because it improves the quality and/or

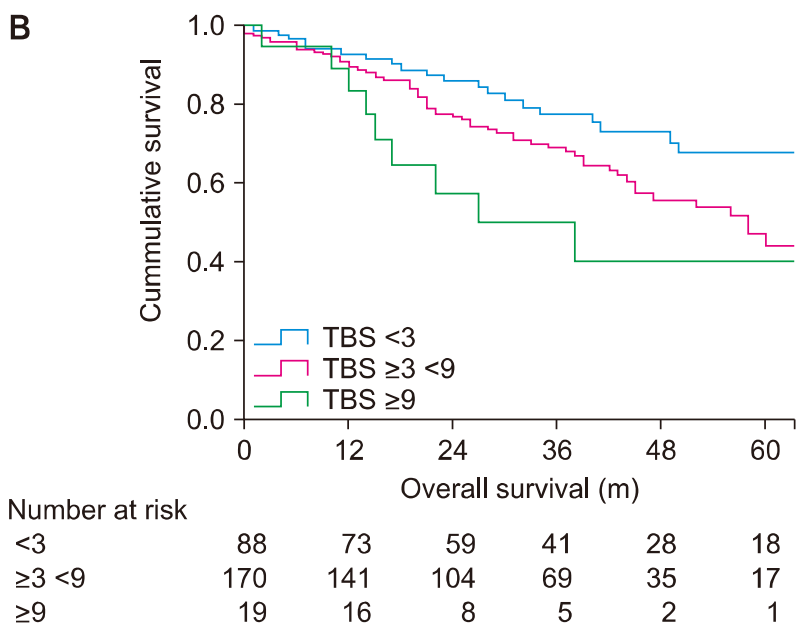

Fig. 2. Kaplan-Meier estimates of overall survival stratified by; (A) age category, $p=0.772$, and (B) tumour burden score, $p=0.010$. 
Table 1. Patient characteristics, tumour and operative data by age group

\begin{tabular}{|c|c|c|c|}
\hline Characteristics & $\begin{array}{l}<75 \text { years } \\
\mathrm{n}=272(\%)\end{array}$ & $\begin{array}{l}\geq 75 \text { years } \\
\mathrm{n}=67(\%)\end{array}$ & $p$-value \\
\hline Gender & & & 0.432 \\
\hline Female & $95(34.9)$ & $20(29.9)$ & \\
\hline Male & $177(65.1)$ & $47(70.1)$ & \\
\hline ASA & & & 0.868 \\
\hline I & $6(2.2)$ & $1(1.4)$ & \\
\hline II & $140(51.5)$ & $33(49.3)$ & \\
\hline III & $126(46.3)$ & $33(49.3)$ & \\
\hline Operation & & & 0.228 \\
\hline Metastasectomy & $127(46.7)$ & $23(34.3)$ & \\
\hline$<3$ segments & $65(23.9)$ & $25(37.3)$ & \\
\hline Hemi-hepatectomy & $62(22.8)$ & $15(22.4)$ & \\
\hline Extended hemi-hepatectomy & $6(2.2)$ & $1(1.4)$ & \\
\hline Stage 1 procedure & $12(4.4)$ & $3(4.5)$ & \\
\hline Total tumours resected ${ }^{\dagger}$ & $2,1-20$ & $2,1-9$ & 0.383 \\
\hline Tumour $\operatorname{size}^{\dagger} / \mathrm{cm}$ & $2.7,0.4-12.6$ & $3.5,0.5-20.0$ & 0.114 \\
\hline $\mathrm{TBS}^{\dagger}$ & $4.12,0.4-20.2$ & $4.32,0.5-20.1$ & 0.463 \\
\hline Margin status & & & $0.002 *$ \\
\hline $\mathrm{R} 0$ & $215(79.6)$ & $63(95.5)$ & \\
\hline $\mathrm{R} 1$ & $55(20.4)$ & $3(4.5)$ & \\
\hline Extrahepatic disease & & & 0.081 \\
\hline No extrahepatic disease & $209(76.9)$ & $58(86.6)$ & \\
\hline Extrahepatic disease & $63(23.1)$ & $9(23.4)$ & \\
\hline Bile duct invasion & & & 0.533 \\
\hline No & $225(83.3)$ & $57(86.4)$ & \\
\hline Yes & $45(16.7)$ & $9(13.6)$ & \\
\hline Vascular invasion & & & 0.801 \\
\hline No & $173(64.1)$ & $41(62.1)$ & \\
\hline Yes & $97(35.9)$ & $25(37.9)$ & \\
\hline Intraoperative blood $\operatorname{loss}^{\dagger} / \mathrm{ml}$ & $400,20-3600$ & $425,30-1800$ & 0.968 \\
\hline Length of stay ${ }^{\dagger} /$ days & $6.5,1-135$ & $7,3-216$ & 0.189 \\
\hline Clavien Dindo score $\geq 3$ & $18(6.6)$ & $4(6.0)$ & 0.847 \\
\hline \multicolumn{4}{|l|}{ 30-day mortality } \\
\hline No & $269(98.9)$ & $66(98.5)$ & 0.791 \\
\hline Yes & $3(1.1)$ & $1(1.4)$ & \\
\hline \multicolumn{4}{|l|}{ 90-day mortality } \\
\hline No & $266(97.8)$ & $65(97.0)$ & 0.554 \\
\hline Yes & $5(1.8)$ & $2(3.0)$ & \\
\hline \multicolumn{4}{|l|}{ Post-operative chemotherapy } \\
\hline No & $108(39.7)$ & $43(64.2)$ & $<0.001^{*}$ \\
\hline Yes & $164(60.3)$ & $24(35.8)$ & \\
\hline
\end{tabular}

*Statistically significant

${ }^{\dagger}$ Median, range

TBS, tumour burden score

quantity of an individual's life. ${ }^{4,8}$ The results of the present study demonstrate no difference in the complication rates or OS of elderly patients undergoing liver resection for CRLM and therefore suggest it is a feasible treatment option for elderly patients.

The disparity between age groups in operative survival, OS and treatment aggressiveness seen by Booth et al. ${ }^{5}$ is not reproduced in our analysis. This could because the treatments used in previous studies are now historical and do not reflect current practice. A recent meta-analysis of CRLM resection in the elderly used 14 studies of which 2 used data collected over 5 years ago but the rest were over 10 years ago. ${ }^{9}$ Regional control of colorectal cancer has improved with the use of $t$ total mesorectal excision 
Table 2. Univariate analysis of overall survival

\begin{tabular}{|c|c|c|c|}
\hline Characteristics & HR & $95 \% \mathrm{CI}$ & $p$-value \\
\hline Age $<75$ years & 1.078 & $0.648,1.791$ & 0.773 \\
\hline Gender & 0.909 & $0.590,1.400$ & 0.664 \\
\hline ASA & & & 0.564 \\
\hline ASA-1 & & & Ref \\
\hline ASA-2 & 0.965 & $0.299,3.112$ & 0.965 \\
\hline ASA-3 & 1.215 & $0.375,3.935$ & 0.745 \\
\hline Operation & & & $0.010^{*}$ \\
\hline Metastectomy & & & Ref \\
\hline$<3$ segments & 0.806 & $0.462,1.405$ & 0.446 \\
\hline Hemi-hepatectomy & 1.642 & $0.984,2.739$ & 0.058 \\
\hline Extended hemi-hepatectomy & 3.297 & $1.004,10.826$ & $50.049^{*}$ \\
\hline Staged procedure & 2.944 & $1.302,6.659$ & $0.010^{*}$ \\
\hline Margin status & 1.421 & $0.835,2.417$ & 0.195 \\
\hline Bile duct invasion & 1.181 & $0.703,1.985$ & 0.530 \\
\hline Vascular invasion & 1.305 & $0.855,1.993$ & 0.217 \\
\hline Blood loss $/ \mathrm{mls}$ & & & 0.641 \\
\hline$<500$ & & & Ref \\
\hline $500-999$ & 1.073 & $0.635,1.812$ & 0.793 \\
\hline 1000-1999 & 1.513 & $0.827,2.768$ & 0.179 \\
\hline$\geq 2000$ & 1.118 & $0.153,8.160$ & 0.912 \\
\hline TBS & & & $0.013 *$ \\
\hline$<3$ & & & Ref \\
\hline$\geq 3<9$ & 1.748 & $1.059,2.886$ & $0.029 *$ \\
\hline$\geq 9$ & 3.054 & $1.388,6.718$ & $0.006^{*}$ \\
\hline Extrahepatic disease & 1.741 & $1.064,2.847$ & $0.027 *$ \\
\hline Post-op chemo & 0.825 & $0.546,1.248$ & 0.363 \\
\hline $\mathrm{CV}<3$ & 1.406 & $0.650,3.045$ & 0.387 \\
\hline Metachronous & & & 0.924 \\
\hline Early metachronous & & & Ref \\
\hline Late metachronous & 0.880 & $0.465,1.667$ & 0.695 \\
\hline Synchronous & 0.903 & $0.497,1.642$ & 0.739 \\
\hline Primary location & & & 0.397 \\
\hline Right colon & & & Ref \\
\hline Left colon & 0.775 & $0.775,1.502$ & 0.450 \\
\hline Rectum & 0.645 & $0.645,1.277$ & 0.208 \\
\hline
\end{tabular}

*Statistically significant

CI, confidence intervals; ASA, american society of anesthesiologists; TBS, tumour burden score; CV, clavien dindo complication score

and chemoradiotherapy. Strategies have become increasingly successful at transforming unresectable disease into resectable disease through; neoadjuvant systemic chemotherapy, radiofrequency ablation of lesions, PVE allowing liver hypertrophy, partial ALPPS or two stage resections. These changes undoubtedly account for increase in survival compared to other studies, even in the elderly group (60 vs. 43 months). ${ }^{9,10}$

A strength of the current study is the use of a recent patient cohort to reflect the contemporary outcomes from
Table 3. Multivariate analysis of overall survival

\begin{tabular}{llcl}
\hline \multicolumn{1}{c}{ Characteristics } & HR & $95 \%$ CI & $p$-value \\
\hline Extrahepatic disease & 1.095 & $0.914,1.311$ & 0.326 \\
TBS & & & 0.104 \\
$\quad<3$ & & & Ref \\
$\geq 3<9$ & 1.439 & $0.922,2.243$ & 0.121 \\
$\geq 9$ & 2.014 & $1.014,3.997$ & $0.045^{*}$ \\
Operation & & & $<0.001^{*}$ \\
Metastectomy & & & Ref \\
$\quad<3$ segments & 1.016 & $0.620,1.664$ & 0.950 \\
Hemi-hepatectomy & 1.557 & $0.971,2.496$ & 0.066 \\
Extended hemi-hepatectomy & 3.957 & $1.637,9.564$ & $0.002^{*}$ \\
Staged procedure & 3.854 & $1.907,7.791$ & $0.000^{*}$ \\
\hline
\end{tabular}

*Statistically significant

TBS, tumour burden score; CI, confidence intervals

liver surgery. This is useful clinical evidence to help patient choices and also shape further developments. Our results represent the experience of a tertiary liver unit working in a centralised cancer network. This is still not the case in many countries with established healthcare systems. Recent data from Germany shows an operative mortality rate of $7.5 \%$ in low volume centres. ${ }^{11}$

The primary outcomes of this study were the low complication rate $(6 \% \mathrm{CV}>3)$ and low operative mortality rate (1.4\% 30-day mortality) in the older patient group. The groups were largely comparable in their patient characteristics', but some differences were noted. Patients underwent similar procedures for the same number and size of tumours but there was an increased R1 resection rate in patients $<75$ years old. This might indeed reflect a more aggressive approach to younger patients with CRLM and possibly a selection bias, but it is difficult to conclude from this data alone.

A limitation of retrospective case series in CRLM is the inability to thoroughly control for the primary disease management. Tertiary referral centres such as ours see patients after heterogenous surgeries, chemotherapy and radiotherapy from a number of referral centres. We did control for post-operative chemotherapy and whilst it was more common in younger patients it did not appear to impact on survival. Anecdotally, we have observed that not all patients completed the planned course of treatment but interestingly Munker et al. ${ }^{12}$ showed that there was no survival difference if the chemotherapy dose is reduced due to side effects. 
The current consensus on post-liver resection chemotherapy for CRLM remains ambiguous but some evidence suggests an impact in high risk patients. ${ }^{13,14}$

The survival analysis clearly showed no difference in OS rates between the two age cohorts and found only type of operation and a high TBS to be independently prognostic. This is an important finding as it should encourage the consideration of liver resection in all patients with CRLM. Patient selection should obviously occur, but this should be done in an MDT environment. We suspect that it is this impartiality that has led to a higher percentage of elderly patients undergoing CRLM resection than in previous studies $(20 \%$ vs. $10-14 \%){ }^{9}$

This study aimed to prove that operating in patients greater than 75 years of age was safe, successful and comparable to operating on those under 75 years. As the incidence of colorectal cancer increases, the aim should not just to get elderly patients through operative treatments more safely, but all patients. Enhanced recovery and prehabilitation schemes are likely to offer further gains here. ${ }^{15,16}$

Patients with resectable disease should be offered surgery and chronological age alone should not be a barrier to this. A pragmatic approach would consider the potential quality and length of life to be gained from curative resection.

\section{ACKNOWLEDGEMENTS}

The authors thank all the staff of Theatre 7, UHW and the Cardiff Liver Unit for all their hard work and commitment. Special thanks to Sian Thomas, Clinical Nurse Specialist, for her enthusiasm and dedication to the patients.

The Preliminary findings were presented at the Association of Surgeons In Training annual conference in Birmingham, UK, March 2020.

\section{CONFLICT OF INTEREST}

The authors have no conflicts of interest to declare.

\section{ORCID}

Nicholas George Mowbray:

https://orcid.org/0000-0001-9931-0145

Carven Chin: https://orcid.org/0000-0003-2301-618X
Patricia Duncan: https://orcid.org/0000-0003-3831-2969

David O'Reilly: https://orcid.org/0000-0002-0959-8315

Zsolt Kaposztas: https://orcid.org/0000-0001-9281-0550

Sameer Junnarkar: https://orcid.org/0000-0003-0588-1014

Nagappan Kumar: https://orcid.org/0000-0001-8324-9685

\section{AUTHOR CONTRIBUTIONS}

Data analysis, original draft: NGM. Data collection, data analysis: CC. Write up review: PD. Write up review: DOR, ZK, SJ. Conceptualization, data analysis, write up and final draft: NK.

\section{REFERENCES}

1. Office for National Statistics. National Population Projections: 2016-based statistical bulletin. Newport: Office for National Statistics; 2018 [cited 2020 Jan 10]. Available from: https://www.ons. gov.uk/peoplepopulationandcommunity/populationandmigration/ populationprojections/bulletins/nationalpopulationprojections/2016 basedstatisticalbulletin.

2. Cancer Research UK. Bowel cancer incidence statistics 2018. London: Cancer Research UK; 2020 [cited 2020 Jan 10]. Available from: https://www.cancerresearchuk.org/health-professional/cancer-statis tics/statistics-by-cancer-type/bowel-cancer/incidence\#heading-One.

3. Engstrand J, Nilsson H, Strömberg C, Jonas E, Freedman J. Colorectal cancer liver metastases - a population-based study on incidence, management and survival. BMC Cancer 2018;18:78.

4. Hackl C, Neumann P, Gerken M, Loss M, Klinkhammer-Schalke M, Schlitt HJ. Treatment of colorectal liver metastases in Germany: a ten-year population-based analysis of 5772 cases of primary colorectal adenocarcinoma. BMC Cancer 2014;14:810.

5. Booth CM, Nanji S, Wei X, Mackillop WJ. Management and outcome of colorectal cancer liver metastases in elderly patients: a population-based study. JAMA Oncol 2015;1:1111-1119.

6. Kumar N, Duncan T, O'Reilly D, Káposztás Z, Parry C, Rees $\mathrm{J}$, et al. Partial ALPPS with a longer wait between procedures is safe and yields adequate future liver remnant hypertrophy. Ann Hepatobiliary Pancreat Surg 2019;23:13-19.

7. Sasaki K, Morioka D, Conci S, Margonis GA, Sawada Y, Ruzzenente A, et al. The Tumor Burden Score: a new "Metro-ticket" prognostic tool for colorectal liver metastases based on tumor size and number of tumors. Ann Surg 2018;267:132-141.

8. Studer P, Horn T, Haynes A, Candinas D, Banz VM. Quality of life after hepatic resection. Br J Surg 2018;105:237-243.

9. van Tuil T, Dhaif AA, Te Riele WW, van Ramshorst B, van Santvoort HC. Systematic review and meta-analysis of liver resection for colorectal metastases in elderly patients. Dig Surg 2019;36:111-123.

10. Cook EJ, Welsh FK, Chandrakumaran K, John TG, Rees M. Resection of colorectal liver metastases in the elderly: does age matter? Colorectal Dis 2012;14:1210-1216.

11. Filmann N, Walter D, Schadde E, Bruns C, Keck T, Lang H, et al. Mortality after liver surgery in Germany. Br J Surg 2019; 106:1523-1529.

12. Munker S, Gerken M, Fest P, Ott C, Schnoy E, Fichtner-Feig1 $\mathrm{S}$, et al. Chemotherapy for metastatic colon cancer: no effect on survival when the dose is reduced due to side effects. BMC 
Cancer 2018;18:455.

13. Mauri D, Filis P, Tsali L, Zarkavelis G, Pentheroudakis G. Role of chemotherapy in resectable liver metastases from colorectal cancer: food for thought from pooled evidence. ESMO Open 2018;3:e00367.

14. Wang Y, Wang ZQ, Wang FH, Yuan YF, Li BK, Ding PR, et al. The role of adjuvant chemotherapy for colorectal liver metastasectomy after pre-operative chemotherapy: is the treatment worthwhile? J Cancer 2017;8:1179-1186.

15. Dunne DF, Jack S, Jones RP, Jones L, Lythgoe DT, Malik HZ, et al. Randomized clinical trial of prehabilitation before planned liver resection. Br J Surg 2016;103:504-512.

16. Melloul E, Hübner M, Scott M, Snowden C, Prentis J, Dejong $\mathrm{CH}$, et al. Guidelines for perioperative care for liver surgery: Enhanced Recovery After Surgery (ERAS) Society recommendations. World J Surg 2016;40:2425-2440. 